\title{
A Gradient Inequality at Infinity for Tame Functions
}

\author{
Didier D'Acunto and Vincent Grandjean
}

\author{
Dipartimento di Matematica \\ Universitàá degli Studi di Pisa \\ Via Filippo Buonarotti 2 \\ 56127 Pisa - Italia
}

dacunto@mail.dm.unipi.it

\author{
Department of Computer Science \\ University of Bath \\ BATH BA2 7AY - England (UK) \\ cssvg@bath.ac.uk
}

Recibido: 17 de enero de 2005

Aceptado: 28 de abril de 2005

\begin{abstract}
Let $f$ be a $C^{1}$ function defined over $\mathbb{R}^{n}$ and definable in a given o-minimal structure $\mathcal{M}$ expanding the real field. We prove here a gradient-like inequality at infinity in a neighborhood of an asymptotic critical value $c$. When $f$ is $C^{2}$ we use this inequality to discuss the trivialization by the gradient flow of $f$ in a neighborhood of a regular asymptotic critical level.
\end{abstract}

Key words: Łojasiewicz inequality, asymptotic critical values, bifurcation values, gradient trajectories, o-minimal structures.

2000 Mathematics Subject Classification: Primary 03C64, 34A26; Secondary 34C08.

\section{Introduction}

Given a $C^{1}$ function $f: U \subset \mathbb{R}^{n} \rightarrow \mathbb{R}$ a Eojasiewicz inequality in a neighborhood of $x_{0} \in \bar{U}$ - the closure of $U$ in $\mathbb{R}^{n}$ for the usual topology - is, for instance, an inequality that quantitatively compares the behavior of $f(x)$ with the one of $|\nabla f(x)|$ or $|x|$ in a neighborhood of $x_{0}$.

When $f$ is analytic and $x_{0}$ is a critical point of $f$, there are at least two well-known such inequalities. The standard Łojasiewicz's gradient inequality (cf. [13]) states that

Supported by the European research network IHP-RAAG contract number HPRN-CT-200100271. 
there exists a smallest rational number $\left.\rho_{f} \in\right] 0,1[$ and a positive constant $C$ such that in a neighborhood of $x_{0}$ we have

$$
|\nabla f(x)| \geq C\left|f(x)-f\left(x_{0}\right)\right|^{\rho_{f}} .
$$

Another important inequality, called Bochnak-Łojasiewicz inequality, states that there is a constant $C_{f}$ such that in a neighborhood of $x_{0} \in \bar{U}$

$$
\left|x-x_{0}\right| \cdot|\nabla f(x)| \geq C_{f}\left|f(x)-f\left(x_{0}\right)\right| .
$$

These inequalities are very useful once we need to deal with quantitative behavior of the function. (These two inequalities are key elements in the proof of the gradient conjecture, see [8].)

The development in the last twenty years of so-called tame geometry, sharing many nice properties with semialgebraic geometry, lead many mathematicians to be interested in this sort of quantitative information about a tame function. One will find more general Łojasiewicz inequalities, for instance, in Pfaffian geometry (cf. [10]) or in the o-minimal structure generated by semialgebraic sets and the exponential function (cf. [11]).

Let us fix the framework of this note. Let $\mathcal{M}$ be a given o-minimal structure expanding the real field (see [5] and [6] for the geometric meaning of this notion and some of its basic and important consequences). In the following by a definable set or a definable function we will mean a set or a function definable in the structure $\mathcal{M}$.

The first contribution of the notion of Łojasiewicz's gradient inequality in the ominimal context was provided by Kurdyka in [7]. In this paper, he was interested in the uniform behavior of the trajectories of the gradient field $\nabla f$, where $f$ is a $C^{1}$ definable function defined over a bounded open subset $U \subset \mathbb{R}^{n}$. To deal with such a question one needs more than just the critical values of $f$. Namely, we say that $c$ is an asymptotic critical value of $f$ if and only if there exists a sequence $\left\{x_{\nu}\right\}_{\nu} \in U$ such that $f\left(x_{\nu}\right) \rightarrow c$ and $\nabla f\left(x_{\nu}\right) \rightarrow 0$. The set of asymptotic critical values is finite and contains the set of critical values and the singular values on the boundary of $U$. We denote by $K_{a}(f)$ the set of asymptotic critical values. To control the behavior of the trajectories of $\nabla f$ in the neighborhood of asymptotic critical fibres Kurdyka established the following key result:

Theorem 1.1 ([7]). If $f: U \rightarrow \mathbb{R}$ is a $C^{1}$ definable function, then for all $c \in K_{a}(f)$ there exist a constant $K_{c}>0$ and a $C^{1}$ definable function $\Psi_{c}:[0,+\infty[\rightarrow \mathbb{R}$ such that $\left|\nabla\left(\Psi_{c}(|f(x)-c|)\right)(x)\right| \geq K_{c}$ for all $x \in U$ and $f(x)$ sufficiently close to $c$.

Now let us assume that $f: \mathbb{R}^{n} \rightarrow \mathbb{R}$ is a $C^{1}$ definable function. Assume the o-minimal structure $\mathcal{M}$ is polynomially bounded.

In [4] we proved that for a value $c$ such that $f^{-1}(c)$ is non compact there exist $C>0$ and a smallest $\rho_{c} \leq 1$ such that for sufficiently large $|x|$ and sufficiently small $|f(x)-c|$ we have

$$
|x| \cdot|\nabla f(x)| \geq C|f(x)-c|^{\rho_{c}} .
$$


In fact we proved this inequality in the semialgebraic context, but the proof extends easily with exactly the same arguments for $C^{1}$ functions defined in polynomially bounded o-minimal structures.

The aim of this note is to prove an analog of inequality (K- $\mathrm{E}$ ) when $\mathcal{M}$ is not polynomially bounded. Such an inequality will be useful to decide whether we can trivialize the function $f$ over a neighborhood of a regular asymptotic critical value $c$ by the gradient field.

Conventions. Let $u$ and $v$ be two continuous functions of a single variable $r$ defined over $\left[1,+\infty\left[\right.\right.$. We will write $w \sim v$ to mean that $u / v$ tends to a limit $l \in \mathbb{R}^{*}$ when $r$ tends to $+\infty$. We will write $u \simeq v$ if $u \sim v$ and $l=1$.

\section{A Bochnak-Łojasiewicz inequality at infinity near an asymp- totic critical value}

Let $f$ be a $C^{1}$ function defined over $\mathbb{R}^{n}$ and definable in $\mathcal{M}$. Let us denote by $\nabla f$ the gradient vector field of $f$ for the standard Euclidean metric.

Definition 2.1. A real number $c$ is an asymptotic critical value of the function $f$ if there exists a sequence $\left\{x_{\nu}\right\}_{\nu} \in \mathbb{R}^{n}$ satisfying the following conditions when $\nu \rightarrow+\infty$

(i) $\left|x_{\nu}\right| \rightarrow+\infty$,

(ii) $f\left(x_{\nu}\right) \rightarrow c$,

(iii) $\left|x_{\nu}\right| \cdot\left|\nabla f\left(x_{\nu}\right)\right| \rightarrow 0$.

Let us denote by $K_{\infty}(f)$ the set of asymptotic critical values of $f$. Let $K_{0}(f)$ be the set of critical values of $f$. Then we recall

Theorem $2.2([2])$. Let $f: \mathbb{R}^{n} \rightarrow \mathbb{R}$ be a $C^{1}$ definable function. The set $K(f):=$ $K_{\infty}(f) \cup K_{0}(f)$ is finite. Moreover, the function $f$ induces a locally trivial continuous fibration over each connected component of $\mathbb{R} \backslash K(f)$.

Unfortunately it is well known, even for a real polynomial function, that the set of bifurcation values of the function can be strictly contained in $K(f)$ (see for instance $f(x, y)=y\left(2 x^{2} y^{2}-9 x y+12\right)$ in [15]). Nevertheless we know that any bifurcation value which is not a critical value is at least an asymptotic critical value (see the works of Némethi and Zaharia in [14] and Loi and Zaharia in [12] to shrink the set of asymptotic critical values where to find the regular bifurcation values).

On the other hand, the attempts to better understand the behavior of the trajectories of the gradient field $\nabla f$ nearby an asymptotic critical level $c$ of $f$, lead us to find a gradient-like inequality at infinity nearby this level for semi-algebraic functions: 
Theorem 2.3 ([4]). Assume that $f$ is semialgebraic. Let $c \in K_{\infty}(f)$. Then there exist a smallest rational number $\left.\left.\rho_{c} \in \mathbb{Q} \cap\right] 0,1\right]$ and a positive constant $K_{c}$ such that

$$
|x| \gg 1 \text { and }|f(x)-c| \ll 1 \Longrightarrow|x| \cdot|\nabla f(x)| \geq K_{c}|f(x)-c|^{\rho_{c}} \text {. }
$$

As a consequence (cf. [4]), if $f$ is $C^{2}$ and $\rho_{c}$ is strictly smaller than 1 , then we can trivialize by the gradient flow of $f$ over a neighborhood of $c$ (and so shrink the set of asymptotic critical values that could be bifurcation values).

Let $\mathbb{R}_{\geq 0}$ be the interval $[0,+\infty[$. In the definable context the first gradient-like inequality at infinity is given by the following

Lemma 2.4 ([3]). Let $f: \mathbb{R}^{n} \rightarrow \mathbb{R}$ be a $C^{1}$ definable function. Let c be an asymptotic critical value of $f$. There exists a continuous definable and non constant function $\left.\Psi_{c}:\right] c-1, c+1\left[\backslash\{c\} \rightarrow \mathbb{R}_{\geq 0}\right.$, such that

$$
|x| \gg 1 \text { and } 0<|f(x)-c| \ll 1 \Longrightarrow|x| \cdot\left|\nabla\left(\Psi_{c} \circ f\right)(x)\right| \geq 1 \text {. }
$$

This inequality can be rephrased as

Lemma 2.5. Under the hypotheses of Lemma 2.4, taking $\nu:] 0,1\left[\rightarrow \mathbb{R}_{\geq 0}\right.$ such that $\nu(t)=\min \left\{\left|\Psi_{c}^{\prime}(c-t)\right|^{-1},\left|\Psi_{c}^{\prime}(c+t)\right|^{-1}\right\}$, we obtain

$$
|x| \gg 1 \text { and }|f(x)-c| \ll 1 \Longrightarrow|x| \cdot|\nabla f(x)| \geq \nu(|f(x)-c|) \text {. }
$$

Since $c \in K_{\infty}(f)$, we necessarily must have that $\nu$ tends to 0 near 0 . The first issue about this inequality is to be able to find the "best" function $\nu$ (i.e., the biggest). The second is, knowing the "best" such function, to be able to have a quantitative information about its asymptotic behavior when we get close to 0 .

For this purpose we will use an elementary lemma about the growth properties of germs at infinity of a single real variable definable functions, which here is an analog of what was done by Kurdyka and Parusiński in [9].

Let us denote by $\mathbb{R}_{\gg 1}$ the germ at infinity of $[1,+\infty[$. Let us recall that if $\varphi$ is the germ of a definable function in a single variable $r$ at infinity, and if $\tilde{\varphi}$ is a

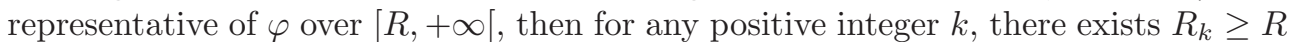
such that $\tilde{\varphi}$ is $C^{k}$ on the interval $] R_{k},+\infty[$, and each derivative is either constant or strictly monotone.

Lemma 2.6. Let $\varphi, \psi: \mathbb{R}_{\gg 1} \rightarrow\left(\mathbb{R}_{>0}, 0\right)$ be non ultimately zero definable functions. Assume that $\varphi>\psi$. Let $K>1$ be given. Then for $r$ large enough we get

$$
\varphi^{\prime}(r) \leq \psi^{\prime}(r) \quad \text { and } \quad K \frac{\psi^{\prime}}{\psi} \leq \frac{\varphi^{\prime}}{\varphi}
$$


Proof. Since $\varphi-\psi$ is a positive function and tends to 0 at $+\infty$ then, by monotonicity the derivative $(\varphi-\psi)^{\prime}$ increases to 0 at infinity, hence we get the first inequality.

Let us first choose $K=p / q>1$ to be a rational number such that $p$ and $q$ are positive and relatively primes. Let $v$ be the definable function defined as $v:=\psi^{p} \varphi^{-q}$. Since $0<v \leq \psi^{p-q}$, we get that $v$ tends to 0 at infinity and thus $v^{\prime} \leq 0$, which provides

$$
\frac{\psi^{p}}{\varphi^{q}}\left[p \frac{\psi^{\prime}}{\psi}-q \frac{\varphi^{\prime}}{\varphi}\right] \leq 0
$$

and thus get the second inequality when $K$ is a rational and by density the proof is over.

Let us come now to the main result of this section, that is, a Bochnak-Łojasiewicz inequality at infinity nearby $c$.

Proposition 2.7. Let $f: \mathbb{R}^{n} \rightarrow \mathbb{R}$ be a $C^{1}$-definable function defined over $\mathbb{R}^{n}$. Let $c$ be an asymptotic critical value of $f$. Then there exist positive constants $R, \varepsilon$, and $K$ such that

$$
\text { if }|x|>R \text { and }|f(x)-c|<\varepsilon \text {, then }|x| \cdot|\nabla f(x)| \geq K|f(x)-c| .
$$

Proof. Assume this does not hold. Then, by the Curve Selection Lemma at infinity there exists a definable curve $\gamma: \mathbb{R}_{\gg 1} \rightarrow \mathbb{R}^{n}$ such that $|\gamma(r)|=r$ and

$$
\lim _{r \rightarrow+\infty} f(\gamma(r))=c \text { and } \lim _{r \rightarrow+\infty} \frac{|\gamma(r)| \cdot|\nabla f(\gamma(r))|}{|f(\gamma(r))-c|}=0 .
$$

Let $v$ be the definable function defined as $v(r)=|f \circ \gamma(r)-c|$.

Under the hypothesis (1) the following holds true.

Lemma 2.8. $r v(r) \rightarrow 0$ when $r \rightarrow+\infty$.

Proof. Assume this is not the case. Hence, there exists a constant $M>0$ such that, ultimately, $v(r) \geq M / r$. Taking the derivatives we obtain

$$
M / r^{2} \leq\left|v^{\prime}(r)\right| \leq\left|\gamma^{\prime}(r)\right| \cdot|\nabla f(\gamma(r))| .
$$

Note that $\left|\gamma^{\prime}(r)\right| \rightarrow 1$ when $r \rightarrow+\infty$. Then there exists $l>0$ such that ultimately

$$
r|\nabla f(\gamma(r))| \geq l / r \geq \frac{l}{M} v(r) .
$$

Hence, $r|\nabla f(\gamma(r))| \cdot v(r)^{-1}$ has a non-zero limit as $r$ tends to infinity. A contradiction. 
We can end the proof of Proposition 2.7. Since $\left|\gamma^{\prime}(r)\right| \rightarrow 1$ as $r \rightarrow+\infty$, there exists a positive constant $M^{\prime}>0$ such that

$$
\frac{|\gamma(r)| \cdot|\nabla(f)(\gamma(r))|}{|f(\gamma(r))-c|} \geq M^{\prime} \frac{r \cdot\left|v^{\prime}(r)\right|}{v(r)}
$$

Let $w(r)=1 / r$. Then

$$
r \frac{\left|v^{\prime}(r)\right|}{v(r)}=\frac{w(r)}{\left|w^{\prime}(r)\right|} \frac{\left|v^{\prime}(r)\right|}{v(r)} .
$$

Since $v$ and $w$ are definable and $v / w \rightarrow 0$ at infinity, for $r$ large enough, $w-v$ is positive and decreases to 0 . From Lemma 2.6, there exists a positive constant $A>0$ such that

$$
\left|\frac{w(r)}{w^{\prime}(r)}\right| \cdot\left|\frac{v^{\prime}(r)}{v(r)}\right| \geq A
$$

which contradicts (1).

Remark 2.9. The proof of Proposition 2.7 is straightforward when $\mathcal{M}$ is polynomially bounded. In this case we can conclude without Lemma 2.6 because for all non ultimately zero definable function $v$ in the single variable $r$, the function $r v^{\prime} / v$ has a non zero limit as $r$ goes to infinity. The reason is that the Hardy field of $\mathcal{M}$ has rank one in this case.

\section{Main result}

Let $f: \mathbb{R}^{n} \rightarrow \mathbb{R}$ be a $C^{1}$ definable function and assume $c \in K_{\infty}(f)$. There is an explicit way to produce the "best" function $\nu$ satisfying Lemma 2.5. For $|x| \gg 1$ and $0<|f(x)-c|=t \ll 1$ let us define the function $m_{c}$ as

$$
m_{c}(t)=\inf \{|x| \cdot|\nabla f(x)|:|f(x)-c|=t\} .
$$

This function is well defined and positive since any such $t$ is a regular value and not an asymptotic critical value. Moreover $m_{c}$ is definable in $\mathcal{M}$, and thus continuous over a small interval of the form $] 0, b]$ and satisfies the inequality of Lemma 2.5.

As a consequence of the inequality of Proposition 2.7, we actually get

Proposition 3.1. Under the previous hypotheses we have:

(i) There exists $A>0$ such that for $0<t \ll 1$ we have $m_{c}(t) \geq A t$.

(ii) If $|x| \gg 1$ and $|f(x)-c| \ll 1$ then $|x| \cdot|\nabla f(x)| \geq m_{c}(|f(x)-c|)$.

(iii) Any definable function $v$ satisfying points (i) and (ii) instead of $m_{c}$ satisfies $m_{c}(t) \geq v(t)$ for all sufficiently small $t>0$. 
Proof. Point (i) is a direct consequence of Proposition 2.7 while point (ii) is just the definition of the function $m_{c}$. Point (iii) comes from the fact that $m_{c}(t)$ is the infimum of the function $|x| \cdot|\nabla f(x)|$ taken on the level hypersurface $f^{-1}(t)$ outside a (given) ball of large radius. A function $v>m_{c}$ would contradict this infimum property.

Remark 3.2. If $\mathcal{M}$ is polynomially bounded, then the function $1 / m_{c}$ of Proposition 3.1 is of the form $1 / m_{c}(r) \simeq C r^{-\rho}$, with $C$ a positive constant and $0<\rho \leq 1$ is an exponent lying in the field of the exponents of the Hardy field of $\mathcal{M}$.

The next consequence is the analog in the current context of [4, Theorem 4.4]. We here assume that $f$ is actually $C^{2}$ and $c \in K_{\infty}(f) \backslash K_{0}(f)$. Then

Theorem 3.3. If the function $1 / m_{c}$ is integrable on an interval of the type $\left.] 0, b\right]$, then we can trivialize the function $f$ over a neighborhood of $c$ by the gradient flow of $f$.

Proof. The proof is adapted from [4, Theorem 4.4]. For simplicity we shall only work with values $t<c$. Let $t_{0}<c$ be such that $\left[t_{0}, c\right] \cap K(f)=\{c\}$ and let $R>0$ be a real number such that Proposition 3.1 holds in $f^{-1}\left(\left[t_{0}, c[) \cap\{|x|>R\}\right.\right.$. Let $x_{0} \in f^{-1}\left(t_{0}\right) \cap\{|x|>R\}$ and let $\gamma$ be a (maximal) trajectory of $\nabla f$ parameterized by the levels of $f$, that is satisfying to the following differential equation

$$
\gamma^{\prime}(t)=\mathbf{X}(\gamma(t)), \quad \text { with initial condition } \gamma\left(t_{0}\right)=x_{0} \in f^{-1}\left(t_{0}\right),
$$

where $\mathbf{X}$ is the vector field $\nabla f /|\nabla f|^{2}$. Thus for all $t$ we obtain $f \circ \gamma(t)=t$.

Integrating equation (2) between $t_{0}$ and $t<c$, we obtain

$$
\int_{t_{0}}^{t} \gamma^{\prime}(s) \mathrm{d} s=\int_{t_{0}}^{t} \mathbf{X}(\gamma(s)) \mathrm{d} s
$$

From equation (3), we get a first inequality

$$
|\gamma(t)| \leqslant\left|\gamma\left(t_{0}\right)\right|+\int_{t_{0}}^{t} \frac{\mathrm{d} s}{|\nabla f(\gamma(s))|}
$$

Using Proposition 3.1, it provides

$$
|\gamma(t)| \leqslant\left|\gamma\left(t_{0}\right)\right|+\int_{t_{0}}^{t} \frac{|\gamma(s)|}{m_{c}(|s-c|)} \mathrm{d} s
$$

Then Gronwall Lemma gives

$$
|\gamma(t)| \leqslant\left|\gamma\left(t_{0}\right)\right| \exp \int_{t_{0}}^{t} \frac{\mathrm{d} s}{m_{c}(|s-c|)}
$$

When $1 / m_{c}$ is integrable, $|\gamma(t)|$ has a finite limit when $t$ tends to $c$. So each trajectory passing through a point of the level $t_{0}$ has finite length between the levels $t_{0}$ and $c$. 
Thus the flow of $\nabla f$ maps injectively the whole level $t_{0}$ into the level $c$. To conclude we use an embedding theorem proved by the first named author [3], stating that any connected component of the level $c$ is injectively mapped into a connected component of the level $t_{0}$ by the flow of $-\nabla f$. Thus the trivialization by the gradient near $c$ is proved.

\section{The Riemannian case}

In this section we assume that $f: M \rightarrow \mathbb{R}$ is a $C^{1}$ definable function defined on a $C^{2}$ definable submanifold $M \subset \mathbb{R}^{n}$ equipped with the definable Riemannian metric $g$ induced by the standard Euclidean metric of $\mathbb{R}^{n}$. We also assume that $M$ is closed, connected, unbounded and without boundary. We respectively denote by $|\cdot|_{g}$ and $\nabla_{g}$ the norm and the gradient with respect to the metric $g$.

In this setting it makes sense to study the function $|x| \cdot\left|\nabla_{g} f(x)\right|_{g}$ and again to define the set $K_{\infty}(f)$ of asymptotic critical values of $f$ with it. It was proved in [2] that $K_{\infty}(f)$ is finite and in [3] that Lemma 2.4 holds with this setting.

In this context, it is easy to verify that the results stated in the present paper are also true. Let $c \in K_{\infty}(f)$. For sufficiently small $t>0$, let $m_{c}$ be the function defined as $m_{c}(t)=\inf \left\{|x| \cdot\left|\nabla_{g} f(x)\right|_{g}:|f(x)-c|=t\right\}$, then the following holds true.

\section{Proposition 4.1.}

(i) There exists $A>0$ such that for $0<t \ll 1$ we have $m_{c}(t) \geq A t$.

(ii) If $|x| \gg 1$ and $|f(x)-c| \ll 1$ then $|x| \cdot\left|\nabla_{g} f(x)\right|_{g} \geq m_{c}(|f(x)-c|)$.

(iii) Any definable function $v$ satisfying points (i) and (ii) instead of $m_{c}$ satisfies $m_{c}(t) \geq v(t)$ for all sufficiently small $t>0$.

Similarly, the main result of this paper extends in this setting as

Theorem 4.2. If the function $1 / m_{c}$ is integrable on an interval of the type $\left.] 0, b\right]$, then we can trivialize the function $f$ over a neighborhood of $c$ by the flow of $\nabla_{g} f$.

\section{References}

[1] J. Bochnak and S. Eojasiewicz, A converse of the Kuiper-Kuo theorem, Proceedings of Liverpool Singularities-Symposium, I (1969/70), 1971, pp. 254-261. Lecture Notes in Math., Vol. 192.

[2] D. D'Acunto, Valeurs critiques asymptotiques d'une fonction définissable dans une structure o-minimale, Ann. Polon. Math. 75 (2000), no. 1, 35-45 (French, with English summary).

[3] Sur la topologie des fibres d'une fonction définissable dans une structure o-minimale, C. R. Math. Acad. Sci. Paris 337 (2003), no. 5, 327-330.

[4] D. D'Acunto and V. Grandjean, On gradient at infinity of semialgebraic functions (2004), 11 pages, preprint. 
[5] L. van den Dries, Tame topology and o-minimal structures, London Mathematical Society Lecture Note Series, vol. 248, Cambridge University Press, Cambridge, 1998.

[6] L. van den Dries and C. Miller, Geometric categories and o-minimal structures, Duke Math. J. 84 (1996), no. 2, 497-540.

[7] K. Kurdyka, On gradients of functions definable in o-minimal structures, Ann. Inst. Fourier (Grenoble) 48 (1998), no. 3, 769-783.

[8] K. Kurdyka, T. Mostowski, and A. Parusiński, Proof of the gradient conjecture of R. Thom, Ann. of Math. (2) 152 (2000), no. 3, 763-792.

[9] K. Kurdyka and A. Parusiński, Quasi-convex decomposition in o-minimal structures. Applications to the gradient conjecture (2001), 28 pages, preprint.

[10] J.-M. Lion, Inégalité de Lojasiewicz en géométrie pfaffienne, Illinois J. Math. 44 (2000), no. 4, 889-900 (French, with English summary).

[11] T. L. Loi, Eojasiewicz inequalities for sets definable in the structure $\mathbf{R}_{\exp }$, Ann. Inst. Fourier (Grenoble) 45 (1995), no. 4, 951-971.

[12] T. L. Loi and A. Zaharia, Bifurcation sets of functions definable in o-minimal structures, Illinois J. Math. 42 (1998), no. 3, 449-457.

[13] S. Łojasiewicz, Ensembles semi-analytiques, I.H.E.S. (1965), preprint.

[14] A. Némethi and A. Zaharia, On the bifurcation set of a polynomial function and Newton boundary, Publ. Res. Inst. Math. Sci. 26 (1990), no. 4, 681-689.

[15] M. Tibăr and A. Zaharia, Asymptotic behavior of families of real curves, Manuscripta Math. 99 (1999), no. 3, 383-393. 